\title{
La modernidad en Valencia a través de tres obras del arquitecto Mauro Lleó.
}

\author{
Martínez Gregori, Carmen \\ Universitat Politècnica de València, Departamento de Composición Arquitectónica, ETS Arquitectura, València, \\ España, carmargr@cpa.upv.es
}

\begin{abstract}
Resumen
Después del estancamiento a nivel urbanístico que supuso la etapa autárquica, comenzó el verdadero "boom" urbano que doblaría el área urbanizada de la ciudad de Valencia. A partir de la solución estructural radioconcéntrica que planteaba el PGOU de 1946, se establecieron los nuevos ejes del desarrollo tanto urbanos como industriales, siendo el del oeste, el de Manises-Quart de Poblet-Aldaia, el especializado en la industria metálica.

Pero el Plan no era viable sin una red de vías radiales que dieran a los caminos históricos la proporción adecuada a su nueva condición. Este es el caso del Camí Reial de Castilla que en el año 1953 se abría al tránsito, convirtiéndose en la nueva entrada de la carretera de Madrid a la ciudad de Valencia y la conexión con el aeropuerto de Manises.

Se creaba así un gran eje comercial e industrial a lo largo del cual se instalarían grandes empresas dadas sus buenas comunicaciones con la capital del Estado. Es el caso de la embotelladora Coca-Cola (1958), de la fábrica de transformados metálicos FLEX (1961) o de la filial de S.E.A.T (1965), edificios todos obra del mismo arquitecto, Mauro Lleó Serret (1914-2001), y que se convirtieron en pioneros de la modernidad en la ciudad.

La planta de embotellar Coca-Cola, aún siendo deudora de los modelos de Gutiérrez-Soto en cuanto a materiales, juega en su composición con volúmenes sencillos de una o dos plantas que macla con acierto relacionando el programa que contienen. La fábrica FLEX es un edificio industrial en altura que muestra en fachada la retícula estructural que lo sostiene que, aunque todavía de hormigón, se confirma como recurso compositivo que va acercando al arquitecto a las soluciones constructivas miesianas y finalmente, el edificio para S.E.A.T. constituyó un paso hacia la construcción estandarizada y la prefabricación, aunque todavía con recelos, en la solución de sus fachadas a base de muros cortina, brise-soleil y marquesinas metálicas.
\end{abstract}

Palabras clave: moderno, industria, hormigón armado, retícula, prefabricación

\begin{abstract}
After town planning stagnation that the autarchic stage supposed, began the real urban "boom" which doubled the urbanized city of Valencia area. From the radio-concentric structural solution posed by the land-use planning of 1946, the new axes of both, urban development and industrial, were established being the west, Manises-Quart de Poblet-Aldaia, specialized in the metal industry.
\end{abstract}

But the plan was not viable without a network of radial roads that gave the historical paths suitable proportion to their new condition. This is the case of Camí Reial de Castilla that in 1953 was opened to traffic, becoming the new entrance road from Madrid to Valencia and the connection to the Manises airport.

A large commercial and industrial axis was created along which large companies given its good communications with the capital of the state would be installed. This is the case of the bottler Coca-Cola (1958), the metal processing factory FLEX (1961) or the subsidiary SEAT (1965), all buildings made by the same architect, Mauro Lleó Serret (1914-2001), that became pioneers of modernity in the city.

Bottling plant Coca-Cola, still being debtor in materials of Gutierrez-Soto models, plays in its composition with simple volumes of one or two plants that join successfully linking the program contained. The FLEX factory is an industrial building height that shows in its façade the structural grid that holds it, although still built with concrete, is confirmed as a compositional resource that is approaching the architect to Miesian constructive solutions. Finally, the S.E.A.T. building was a step towards standardized construction and prefabrication, though still distrusts in the solution of its façades built with curtain walls, brise-soleil and metal canopies. Highlighting the exhibition vehicle building, a geodesic sphere of steel and glass.

Key words: modern, industry, reinforced concrete, grid, prefabrication 


\section{Introducción}

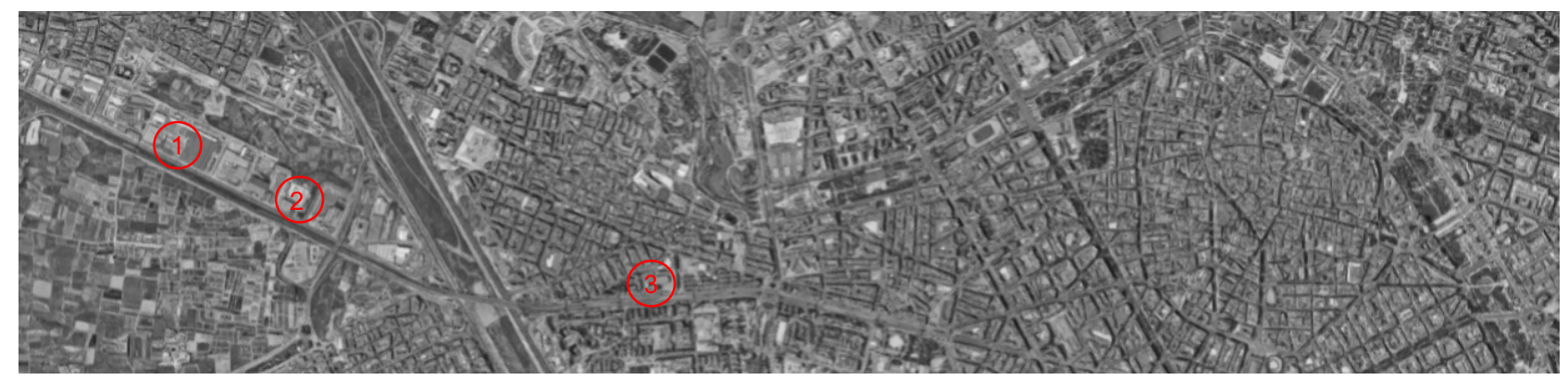

Fig. 1. Fotografía aérea de la ciudad de València realizada en el año 2010. valencia.es [consulta: 10 de febrero 2017]. Disponible en: http://mapas.valencia.es/WebsMunicipales/urbanismo/web urbanismo.jsp?lang=es\&lang=1\&nivel=5\%5f6 1.Planta de embotellar "Coca-Cola", 2.Fábrica de Transformados Metálicos FLEX, 3. Filial de S.E.A.T. en Valencia

Una vez superado el estancamiento urbano de la etapa autárquica entre los años 1939 y 1957, se inició en Valencia un gran crecimiento urbanístico que doblaría su área urbanizada mediante un modelo tentacular orientado hacia la economía terciaria.

En la década de los cuarenta se creó la Comisión Superior de Ordenación de la provincia, concretamente en 1944, y tuvo como encargo inicial la redacción de un plan de ámbito provincial, que finalmente no se tramitó pero serviría para la redacción de un Plan General de Ordenación Urbana de la ciudad que se aprobó finalmente el 18 de diciembre de 1946.

Este Plan General afectaba a la mayoría de los municipios de la comarca, que se ponían así al servicio de la ciudad central a partir de un modelo radiocéntrico sobre el que se situaban los nuevos ejes de crecimiento urbano: dos de ellos residenciales, hacia Burjassot-Godella y Torrent, y tres industriales, al norte el textil-químico, al oeste la industria metálica y ceramista de Manises-Quart de Poblet-Aldaia y al sur el maderero. Se delimitaban pues diferentes sectores con una funcionalidad concreta dejando entre ellos las zonas de tolerancia industrial (la ronda y el Camino de Tránsitos), primero y tercer anillo de ensanche a partir del núcleo antiguo y las zonas de preferencia industrial al este y noreste de la ciudad.

Pero el Plan no era viable sin una ampliación de la red viaria y por ello se abrieron nuevas vías radiales y se desdoblaron los recorridos históricos saturados y congestionados, como el Camí Reial de Castilla, que además, se propuso desviar ${ }^{1}$.

El año 1953, la avenida de Castilla, completamente pavimentada, se abría al tránsito. Se convertía en la nueva entrada de la carretera de Madrid a la ciudad de Valencia como prolongación de las calles del Pintor Benedito, San José de Calasanz y San Francisco de Borja. El proyecto tenía una anchura de cuarenta metros desde el Camino de Tránsitos al cruce con el tren de Lliria y desde allí hasta la carretera de Torrent sesenta y cinco, aunque durante un tiempo, la avenida tuvo tan solo diez metros de ancho con zonas laterales para paseo o circulación de carros, quedando el aeródromo de Manises tan solo a nueve kilómetros del centro ${ }^{2}$. Se creaba así un gran eje comercial e industrial a lo largo del cual se instalarían grandes empresas dadas sus buenas comunicaciones con la capital del estado, Madrid.

La década entre 1960 y 1970 es el período en el que el crecimiento urbano y demográfico de la ciudad se extendió con rapidez a otros núcleos de L'Horta y se podría afirmar que es cuando se creó efectivamente el Área Metropolitana de València. La ciudad quedó como centro de servicios, coordinadora de toda el área, y propició la descentralización de la actividad industrial en los núcleos periféricos que hubo que delimitar, pues atrajeron a gran número de población inmigrante. De estos núcleos fue el de L'Horta Oest (Manises-Quart de Poblet-Aldaia) el que experimentó mayor crecimiento fruto de su actividad industrial, pues ya contaba con un sustrato artesanal lo que facilitó el desarrollo de polígonos industriales.

\footnotetext{
${ }^{1}$ GAJA DÍAZ, F. y BOIRA MAIQUES J. V. Planeamiento y realidad urbana en la ciudad de Valencia (1939-1989). Cuadernos de Geografía. 1994, 55, 63-89
}

${ }^{2}$ La avenida de Castilla se abre al tránsito. Las Provincias 1953 [en línea] valenpedia.lasprovincias.es [consulta: 10 de agosto 2014]. Disponible en: http://valenpedia.lasprovincias.es/historia-valencia/1953/la_avenida_de_castilla_se_abre_al transito 
La utilización generalizada del automóvil en 1966 también influyó en el concepto de la "Gran Ciudad" de forma que el tráfico aumentó en las grandes vías viniendo de los accesos de Madrid o Barcelona, pero también del entorno metropolitano más próximo. Se podría afirmar por tanto, que la industrialización y la transformación del sistema de transportes terrestres, vinculada directamente al automóvil, influyeron de forma conjunta en el proceso de modernización económica de la ciudad y su área metropolitana.

En lo referente a lo económico, el fin de la Guerra Civil abrió paso a una etapa marcada por el aislacionismo. El periodo que transcurrió entre 1939 y el Plan de Estabilización del verano de 1959, representó la implantación en España de un modelo de industrialización basado en una intensa intervención y regulación por parte del Gobierno, con altas barreras comerciales que cerraron el mercado interno a la competencia exterior.

El crecimiento de la producción respondió en estos años al esquema conocido como industrialización por sustitución de importaciones, lo que impuso una actividad contraria a la exportación en el conjunto de la actividad económica. Este cierre de la economía española, que se vio restringida a sus recursos propios y a su mercado interno, es lo que caracterizó en mayor medida esta etapa de dos décadas conocida como la autarquía.

Para la industria valenciana los años cuarenta y cincuenta fueron muy difíciles. En primer lugar ello se debió a la fuerte contracción de los mercados de consumo que siguió al fin de la guerra. Pero no solamente la destrucción material y las dificultades de abastecimiento de materias primas y de energía frenaron la recuperación de la economía sino que, la continua pérdida de poder adquisitivo de los salarios limitó enormemente la expansión del mercado. En segundo lugar, las ventas al exterior fueron perdiendo atractivo a medida que el tipo de cambio de la peseta se hacía más irreal, habiendo sido aconsejable una importante devaluación. La inversión se orientó al ahorro de las clases pudientes de la población que invirtieron en propiedad rústica y urbana en detrimento de la inversión industrial.

En cuanto a las iniciativas industriales oficiales, procedentes del Instituto Nacional de Industria (en adelante INI), hay que decir que afectaron en muy escasa medida a la Comunitat Valenciana. Una estimación indica que en 1955 tan solo el 2,5\% del inmovilizado total del INI se ubicaba en la región ${ }^{3}$. Debe citarse en todo caso la instalación de la empresa pública Elcano para la producción de motores y máquinas auxiliares para buques, que abrió sus puertas en Manises en 1951.

A partir de 1953 comenzó una recuperación de la actividad industrial aunque, el cambio de orientación en el modelo de crecimiento de la economía española tuvo su punto de partida definitivo en el Plan de Estabilización Económica de 1959 que frenó la inflación, devaluó la peseta y fue seguido de medidas liberalizadoras de las importaciones.

En el contexto internacional, el momento, además, era favorable para que un país de nivel intermedio-bajo de desarrollo se abriera al exterior, ya que las principales economías europeas, y también los Estados Unidos, y Japón, vivían el período más prolongado e intenso de crecimiento económico de la historia y podían ejercer un importante efecto de arrastre sobre la economía española a través del comercio y la inversión directa ${ }^{4}$.

Y así es como creció urbanística y económicamente el eje este-oeste que vinculaba la ciudad de València con Madrid, convirtiéndose en un potente eje a nivel de comunicaciones gracias a su situación estratégica pero también, en una gran plataforma publicitaria donde se situaban las marcas más importantes dentro del sector industrial y comercial del momento. Entre estas encontramos la embotelladora Coca-Cola (1958), la fábrica de transformados metálicos Flex (1961) y la filial de Seat (1965), edificios todos ellos obra del mismo arquitecto, Mauro Lleó Serret (1914-2001), y que se convirtieron en pioneros de la modernidad en la ciudad.

Mauro Lleó se tituló en la Escuela de Arquitectura de Madrid en el año 1940, una vez finalizada la Guerra Civil. Durante los primeros años de profesión ligados a la posguerra, se vinculó a la Dirección General de Regiones Devastadas trabajo que compaginó formando parte del equipo técnico de la Comisión Superior del Plan de Ordenación de la provincia de València, que derivaría en la Corporación Administrativa Gran València, y con trabajos profesionales de edificación que desarrollaba desde su propio despacho.

Si bien sus inicios en la edificación se vinculan a una arquitectura academicista y tradicional, será su segunda etapa profesional, la que se inicia a mediados de los cincuenta, la que le acercará a la modernidad con edificios

\footnotetext{
${ }^{3}$ MARTÍN ACEÑA, P. y COMÍN COMÍN, F. La acción regional del Instituto Nacional de Industria. NADAL OLLER J. y CARRERAS I ODRIOZOLA A. (dir.) Pautas regionales de la industrialización española: siglos XIX y XX. Barcelona: Ariel, 1990, p. 379-420

${ }^{4}$ REIG MARTÍNEZ, E. Historia breve de la industria valenciana. Dos siglos de industrialización en la Comunitat Valenciana. Valencia: Colegio Oficial de Ingenieros Superiores Industriales de la Comunitat Valenciana, 2007, pp.27-63.
} 
que tenderán hacia la prefabricación y la estandarización de soluciones.

Esta etapa es la más fructífera del arquitecto con numerosos proyectos vinculados a distintos campos como la edificación residencial, la industrial, la arquitectura efímera o el interiorismo, pero también encargos de rehabilitación y urbanismo. Los tres edificios que se comentan a continuación: la planta de embotellar Coca-Cola, la fábrica de transformados metálicos Flex o la filial Seat de València, pertenecen al campo industrial, ya que es el que más rápidamente asume los principios sobre los que se basa la modernidad, a diferencia de la arquitectura residencial sujeta a la opinión siempre más conservadora de sus usuarios.

Cabe decir que de los edificios que se comentan, solo uno de ellos aparece en la base de datos del Registro DOCOMOMO: la filial Seat de València. Sin embargo, mucha de su obra ha sido publicada en bibliografía especializada como en el libro 20x20. Siglo XX, veinte obras de arquitectura moderna o La arquitectura de la industria, 1925-1965. Registro DO.CO.MO.MO. Ibérico, entre otros.

\section{Proyecto de planta de embotellar "Coca-Cola" en Quart de Poblet, Valencia (1958)}

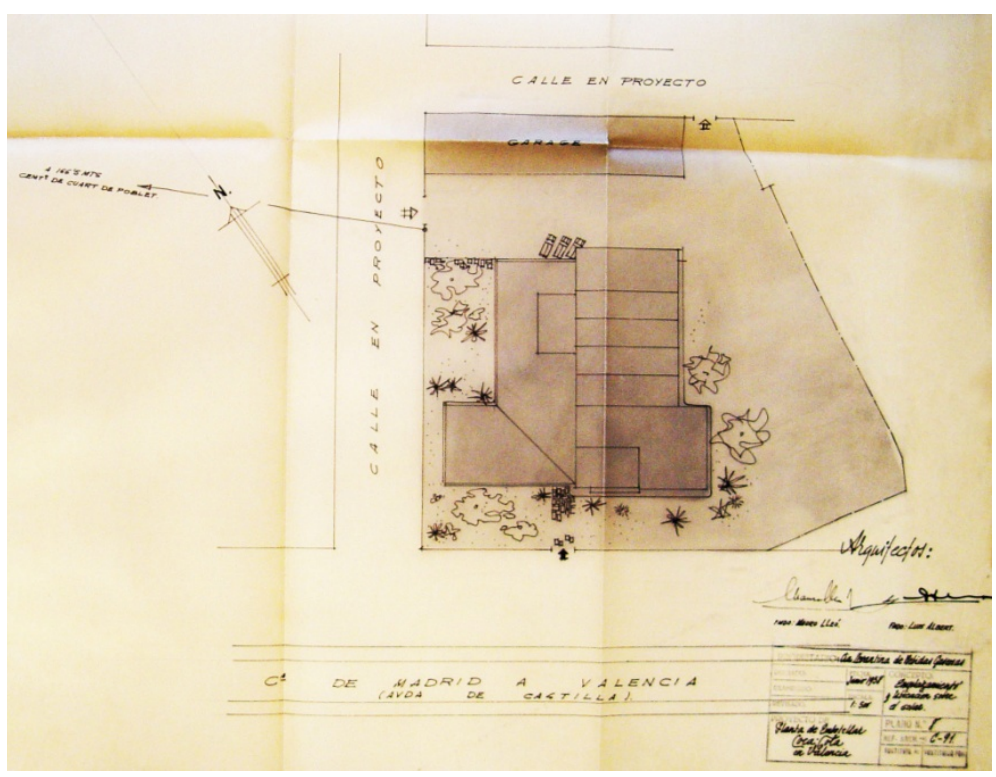

Fig. 2. Plano de situación correspondiente al proyecto de Planta de embotellar "Coca-Cola”. Junio de 1958.

Mauro Lleó firmó el proyecto inicial de planta embotelladora el año 1958, el único en colaboración con Luis Albert, y las sucesivas ampliaciones hasta marzo de 1971, fecha de la quinta y última ampliación que diseñó para la empresa.

Los arquitectos, siguiendo los preceptos modernos, hicieron una separación entre el tráfico rodado y el peatonal, premisa en la que coincidirían los tres proyectos que se presentan. El tráfico peatonal lo situaban en la fachada principal, tras un jardín de formas sinuosas que combinaba distintos materiales junto a pequeñas láminas de agua y riachuelos que descansaban sobre un tapiz verde que todo lo unificaba, con la voluntad de querer recrear el campo en un entorno industrial y con una gran cantidad de tráfico del que buscaban, con toda seguridad, protegerse. El resto de accesos a la planta, como los de servicio para camiones, se resolvían por las calles perpendicular y paralela a la avenida de forma que no se cruzaban el acceso peatonal y el acceso rodado principal con el acceso rodado de servicio. Además, aparecía también en la planta una calle interior que separaba el edificio principal de la nave de talleres, quedando espacio suficiente para el aparcamiento de camiones.

Los espacios del proyecto obedecían al programa funcional que se entregó a los arquitectos con el encargo y contemplaba zonas de producción, ventas, administración, oficinas generales y dirección, y visitas o propaganda (Fig. 3). Así, la planta embotelladora estaba formada inicialmente por dos edificios, uno de ellos el principal que reunía las zonas descritas y un segundo edificio de talleres que no aparecía grafiado en los planos de planta del proyecto. 

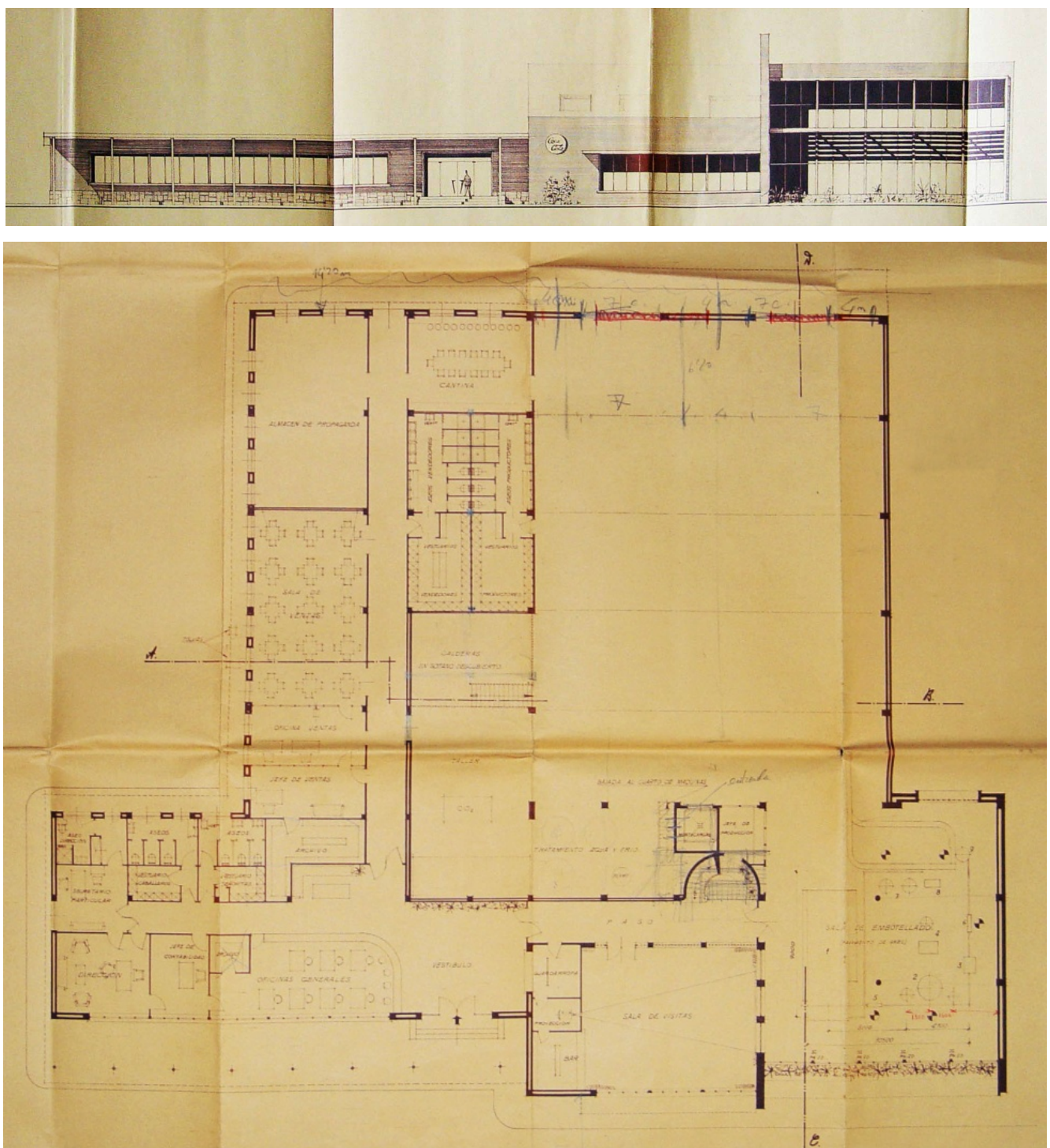

Fig. 3. Fachada sur y planta baja del edificio principal según proyecto de Planta de embotellar Coca-Cola. Junio de 1958.

El edificio principal, aún siendo deudor de los modelos de Gutiérrez-Soto en cuanto a materiales de acabado como el ladrillo caravista y el gresite, jugaba en su composición con volúmenes sencillos de una o dos plantas que maclaba con acierto relacionando el programa que contenía cada uno de ellos.

El primer volumen era un cuerpo en forma de $L$ invertida de una sola planta, cerrado, con una altura libre interior de tres metros y con un porche de acceso que ocupaba todo su frente principal a la avenida con una profundidad de cuatro metros. En su interior, el programa se organizaba en distintas zonas que comprendían un gran vestíbulo de recepción y oficinas, zona de servicios, vestuarios, cantina, almacén de propaganda y despachos de jefes de venta, contabilidad y dirección. 

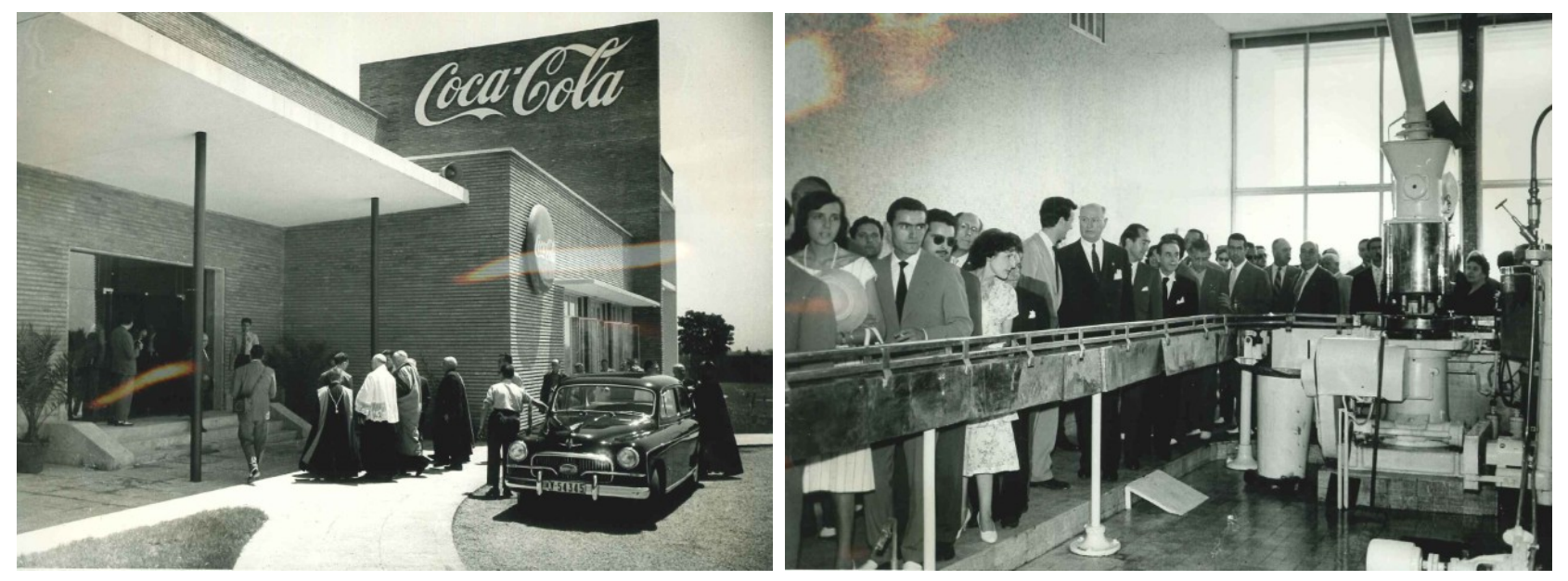

Fig. 4. Fotografía de la inauguración de la fábrica Coca-Cola en Valencia. Acceso principal y sala de embotellado.

En segundo lugar, un paralelepípedo de dos plantas que contenía la zona de producción y visitas y que a su vez se maclaba con la nave de almacenamiento. En la fachada principal este volumen quedaba dividido prácticamente en dos partes iguales por un muro que sobresalía tanto en planta como en altura, dando importancia y presencia al espacio donde se producía la Coca-Cola. Era un elemento que actuaba de reclamo donde, además, se colocaba el logotipo de la marca para hacerlo visible para los vehículos que accedían a la capital desde la carretera de Madrid. (Fig. 4). En su interior, la sala de visitas, la sala de embotellado, el despacho del jefe de producción y la zona de tratamiento de agua y frío en planta baja y en planta primera, el almacenaje de los distintos productos necesarios para la fabricación de la Coca-Cola, completaban el programa.

Por último, la nave de almacenamiento, de forma paralelepipédica de veintitrés metros de luz, formada por cinco crujías de seis con veinticinco metros resueltas con una cubierta en forma de diente de sierra. Esta dejaba unas aberturas orientadas a norte para la iluminación natural de la nave que se resolvían con una carpintería metálica fija cerrada con vidrio armado, recurso que se convertiría en seña de identidad de todos los proyectos industriales del arquitecto.

La construcción presentaba distintos tipos de aberturas y soluciones en función de la orientación y de la representatividad de la fachada. Así pues, en la fachada principal sur el edificio se abría al visitante y a la vía de acceso a la ciudad mostrando su interior con grandes acristalamientos que invitaban a entrar. Dada la orientación de esta fachada, la sala de embotellado se protegía del soleamiento con una "persiana fija horizontal de aluminio", tal y como decía la memoria del proyecto, otro de los recursos que caracterizaría los edificios de Mauro Lleó, tanto docentes como industriales (Fig. 4). Además, esta fachada, retranqueada dos metros con respecto a la línea marcada por la sala de visitas, resolvía este espacio con un ajardinamiento que no impedía la visión de lo que ocurría en el interior pero protegía de un excesivo acercamiento.

Toda la estructura del edificio se resolvió con hormigón armado salvo la cubierta en diente de sierra de la nave de almacenamiento y los pilares del pórtico de acceso y huecos de fachada, los primeros resueltos con perfiles de acero estirado en frío de cinco pulgadas de diámetro interior y los últimos mediante dos UPN 140.

\section{Proyecto de edificio para fábrica de transformados metálicos, Flex, en Quart de Poblet, Valencia (1961)}

En un solar cercano a la planta embotelladora, se construyó el nuevo edificio para fábrica de transformados metálicos. Esta construcción ocupaba parte del mismo previendo un desarrollo crecedero de la industria con posibles futuras ampliaciones por el norte y por el oeste, dejando un espacio ajardinado de protección frente a la avenida al igual que ocurriera en la planta de embotellar Coca-Cola. 


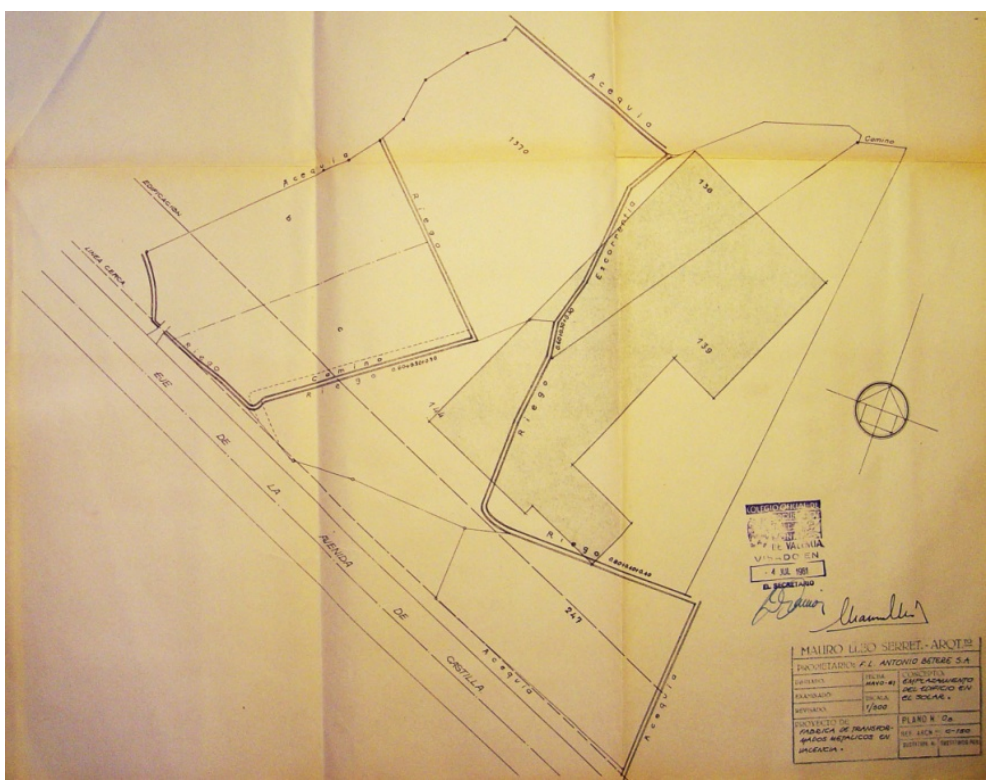

Fig. 5. Plano $\mathrm{n}^{\circ}$ OB. Emplazamiento del edificio en el solar. Proyecto de Fábrica de Transformados Metálicos en Valencia. Mayo de 1961.

El conjunto fabril proyectado por Mauro Lleó, consta de tres volúmenes completamente diferenciados (Fig. 6). El edificio principal, de cinco plantas de altura y forma rectangular, es el volumen más alto del conjunto. Construido con una estructura de hormigón armado a base de pilares y vigas de cuelgue, el arquitecto empezaba a mostrar en este la retícula estructural como recurso compositivo en fachada que cobraba protagonismo al estar situada en el plano exterior de dicho volumen. En su orientación principal sur, el espacio entre la fachada interior y la retícula de pilares, no accesible desde las plantas superiores, quedaba ocupado por unas lamas horizontales de hormigón armado fabricadas "in situ". Estas ayudaban a proteger del soleamiento la fachada interior que se resolvía en cada planta con un murete de caravista, de distinta altura en función del nivel, y acristalamiento cerrando el resto de la altura hasta el forjado superior (Fig. 7).

Este edificio estaba pensado para trabajos delicados de poca carga organizados verticalmente de arriba hacia abajo, siendo la planta baja la destinada a exposición, oficinas, archivo y dirección, con un acceso directo a la zona de venta en su fachada recayente a la Avenida de Castilla. Las plantas altas, de primera a quinta, eran diáfanas, iluminadas y ventiladas por tres de sus fachadas (este, oeste y la principal sur), y todas ellas estaban destinadas a la fabricación de carcasas. Llama la atención la organización del trabajo en vertical recordando las hilanderas inglesas del siglo XIX.

El resto de cerramientos de este volumen, y aun necesitando protección solar en algunas orientaciones como la oeste, se resolvían en línea de fachada, de nuevo mostrando la retícula de hormigón armado de la estructura del edificio, con paños de ladrillo caravista y ventanas en su parte alta cerrando los huecos que dejaba la estructura.

El siguiente volumen era una nave de una sola planta destinada a talleres de forja y mecanización y almacenes metálicos. Este edificio estaba cubierto con una estructura metálica en forma de dientes de sierra que generaba unas aberturas orientadas a norte para la iluminación natural de la nave, por lo que su cerramiento de caravista era totalmente ciego. De nuevo el recurso de la iluminación a norte en la cubierta caracterizando el edificio.

Por último, el conjunto tiene un tercer bloque, un paralelepípedo de cuatro plantas destinado a servicios y viviendas, que soluciona su fachada sur con el mismo recurso que el edificio principal, manteniendo la retícula estructural de hormigón armado en primer plano y retranqueando el cerramiento con respecto a esta. Sin embargo, hay una clara voluntad del arquitecto por dar el máximo protagonismo al edificio principal industrial de forma que este se adelanta con respecto al de viviendas, generando una sombra que aumenta visualmente la distancia entre ellos (Fig. 6). 

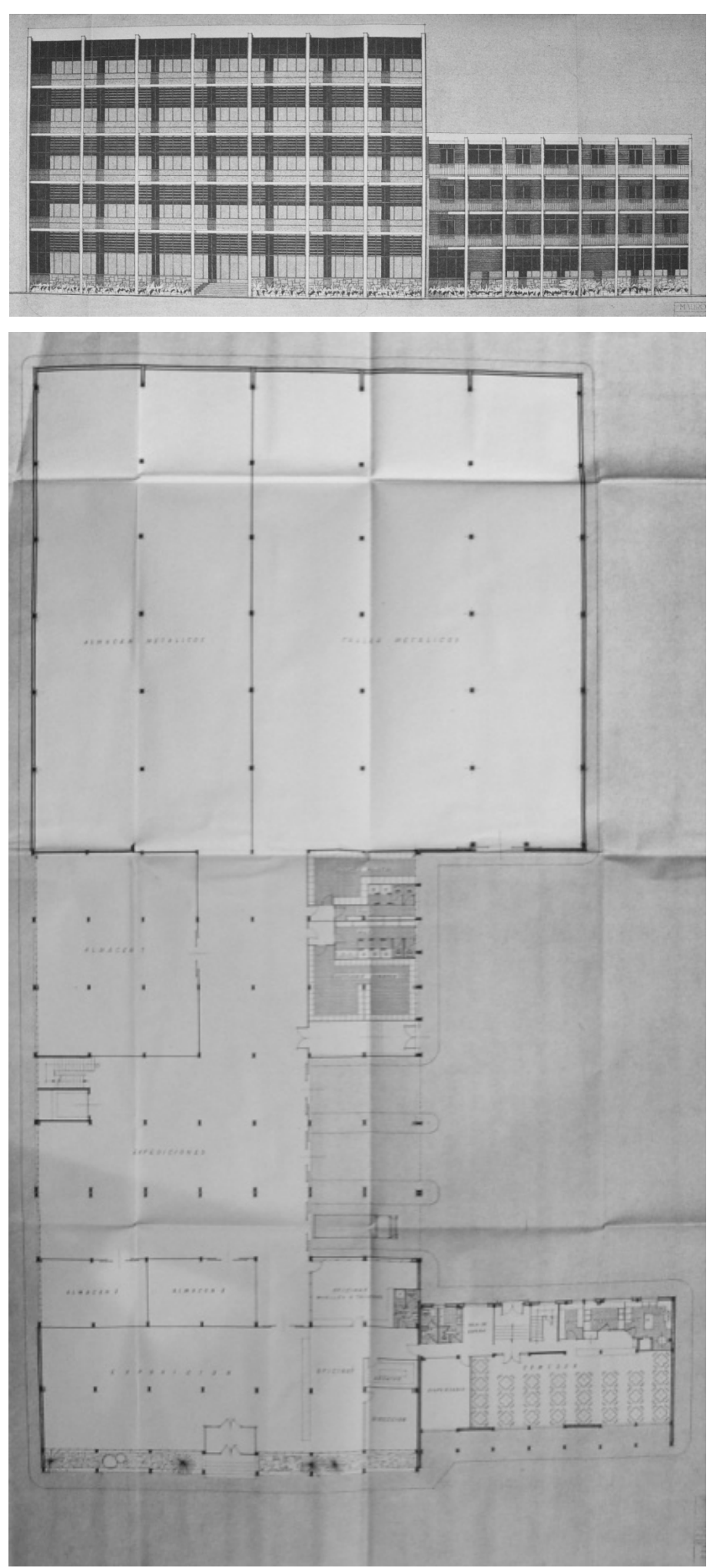

Fig. 4. Plano $n^{\circ} 5$, modificado, fachada sur y plano $n^{\circ} 2$, planta baja. Proyecto de Fábrica de Transformados Metálicos. Mayo de 1961. 


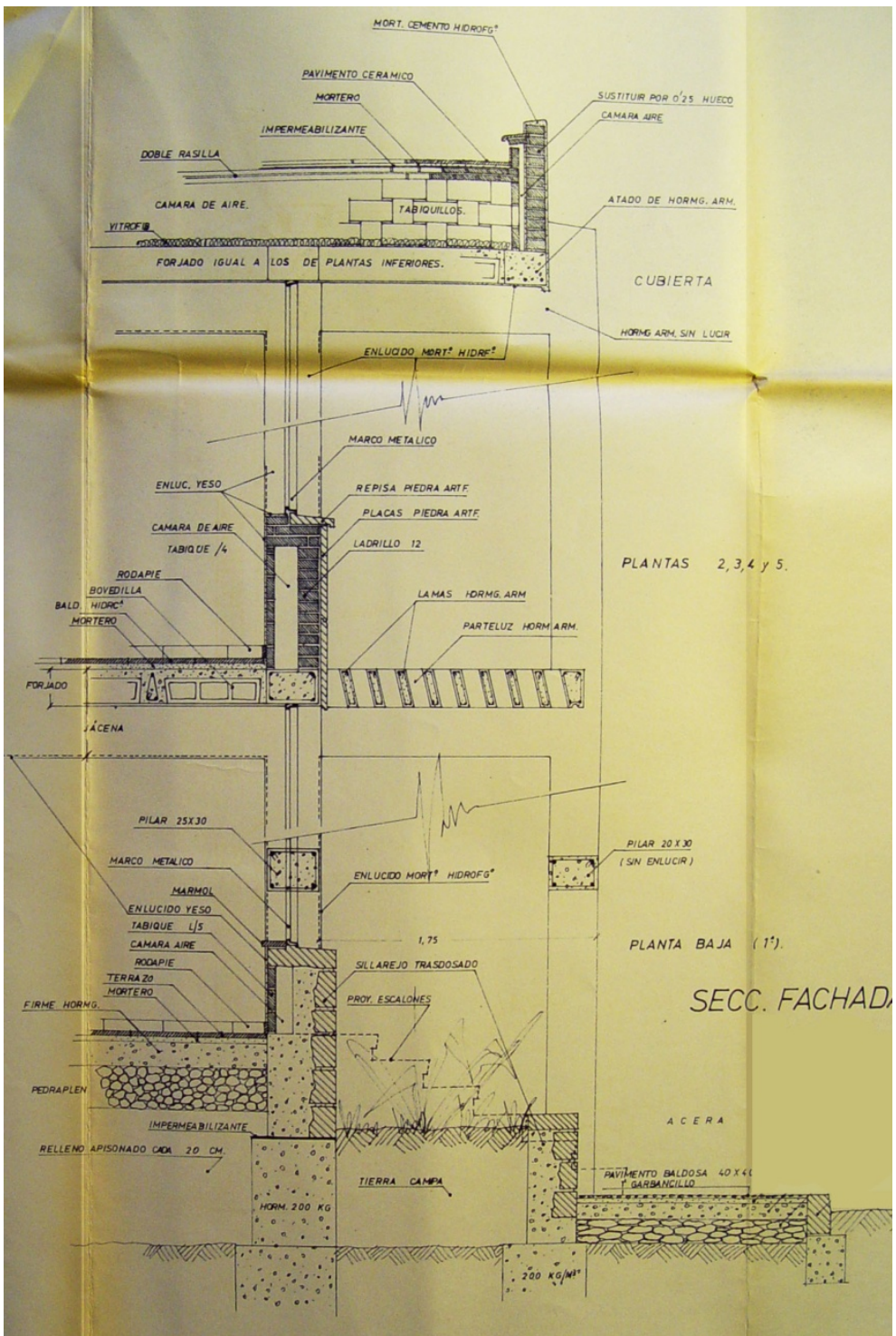

Fig. 7. Plano $n^{\circ}$ 18, detalle de fachada sur del Proyecto de Fábrica de Transformados Metálicos en Valencia. Mayo de 1961.

La estructura principal de la fábrica es de hormigón armado por dos razones fundamentales, por la facilidad de una ejecución inmediata y por su mayor resistencia al fuego. Dadas las dimensiones del conjunto, se debían situar tres juntas de dilatación que claramente se aprecian tanto en planta como en fachada. Dos de ellas se correspondían con la separación entre los distintos bloques: entre el edificio principal y la nave de almacenamiento, y entre el edificio principal y el edificio de servicios y viviendas. La tercera, sin embargo, estaba situada en el edificio principal, aproximadamente en su punto medio.

Conviene destacar la estructura reticular del edificio principal compuesta por pórticos perpendiculares a la fachada sur, con un intereje de cuatro con sesenta metros, mientras que la luz entre pilares era de cinco con cincuenta metros. Esta separación era según los ingenieros de la industria, "lo ideal para la clase de trabajo a realizar". Los forjados se proyectaron de losa nervada con bovedillas de hormigón armado vibrado como encofrado perdido que, tal y como comentaba la memoria, podrían ser sustituidas por un sistema similar prefabricado como sería el Pratton. Por tanto, aunque todavía de hormigón, la retícula se confirma como recurso compositivo que va acercando al arquitecto a las soluciones constructivas miesianas (Fig. 7) con la voluntad, además, de sustituir en lo posible los sistemas constructivos de forjados de montaje "in situ" por otros prefabricados que habrían mejorado los plazos de ejecución. 


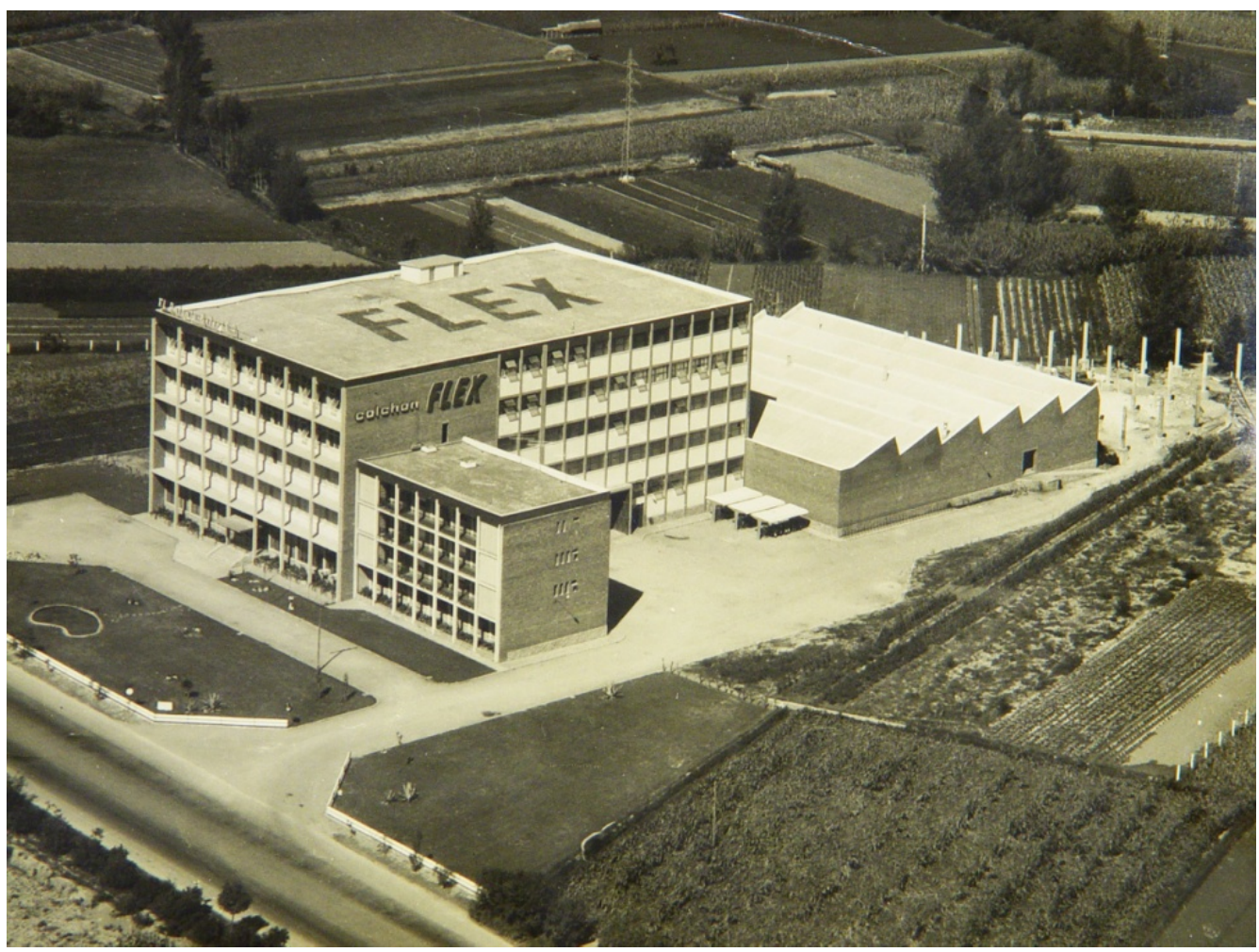

Fig. 8. Imagen del edificio recién iniciada la ejecución de la ampliación de la nave en el año 1963.

\section{Proyecto de edificio para la filial de S.E.A.T. (Sociedad Española de Automóviles de Turismo, S.A.) en Valencia (1965)}

Mauro Lleó recibió el encargo de proyectar y dirigir la obra del edificio para la filial de la Seat en Valencia en 1963. El proyecto, redactado finalmente dos años más tarde, constituiría el paso hacia la construcción estandarizada y la prefabricación, aunque todavía con recelos, en la solución de sus fachadas a base de muros cortina, brisesoleil y marquesinas metálicas.

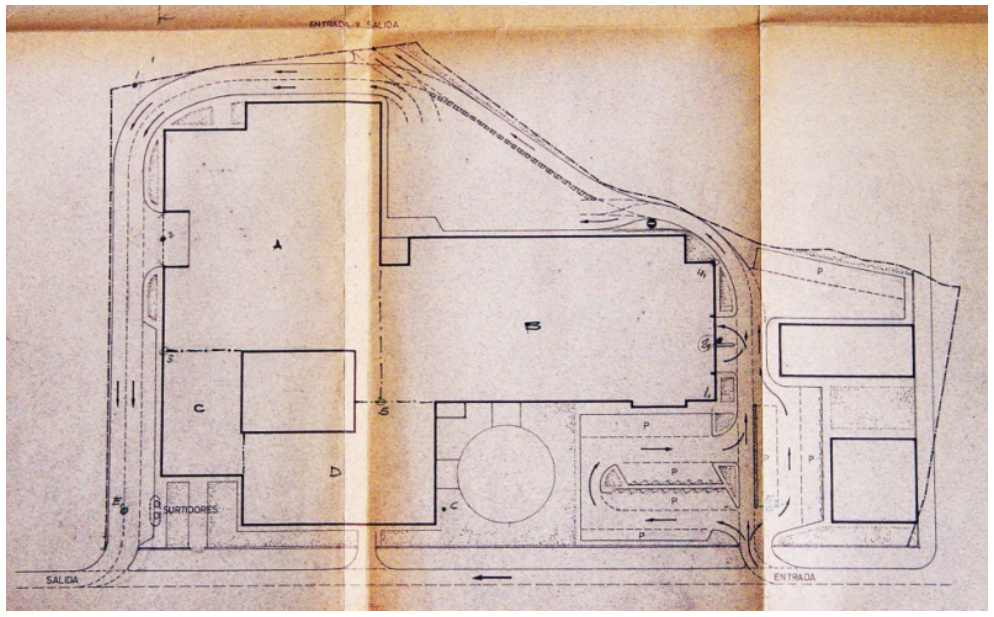

Fig. 9. Plano de urbanización del anteproyecto Filial S.E.A.T. en Valencia. Marzo de 1964. 
Compositivamente el edificio respondía al módulo de seis con cuarenta metros que la empresa marcaba como premisa para todos sus proyectos, distribuyendo alrededor de un patio interior cinco edificios $(A, B, C, D$ y E) que resolvían cada uno de ellos, con su altura y dimensión, una parte del programa dado al arquitecto. Entre estos, destacaba volumétricamente el edificio de taller de nuevo por la solución de su cubierta en diente de sierra, y el edificio de exposición de vehículos, que finalmente no se construyó, una esfera geodésica de acero y vidrio, "un elemento muy singular de atracción, un poliedro de cristal rodeado de jardín y únicamente unido por elementos muy ligeros de una sola planta", tal y como explicaba el arquitecto en una carta al Sr. Alcalde Presidente del Excelentísimo Ayuntamiento de Valencia en respuesta a la denegación de concesión de licencia de obras para la Filial en 1965. Un dibujo del arquitecto del conjunto del edificio, serviría para su presentación ante la propiedad adelantando la imagen de lo que sería el proyecto una vez ejecutado (Fig. 10).

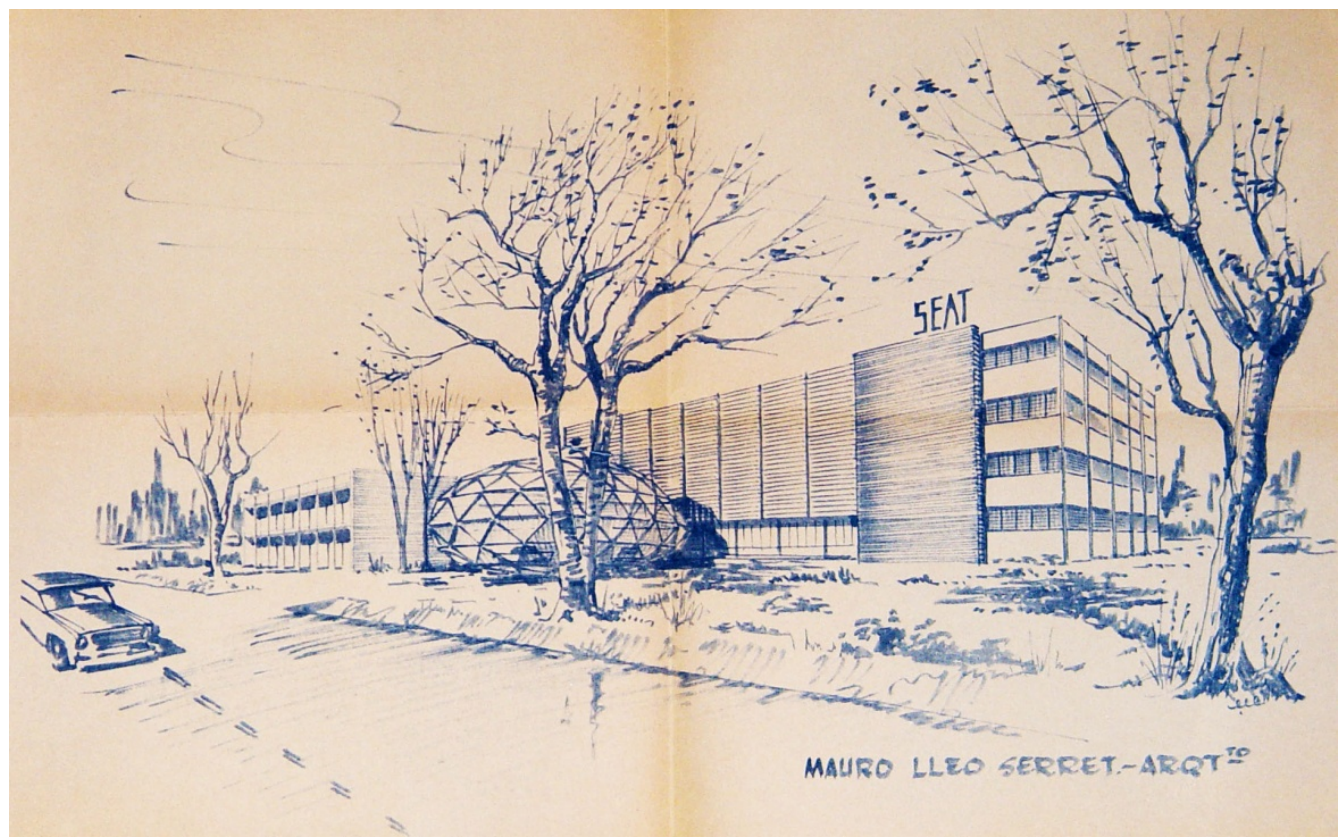

Fig. 10. Perpectiva anteproyecto Filial Seat en Valencia. 1964.

El edificio A consta de dos plantas. La primera o baja se destinaba en su parte noroeste a acceso de personal obrero, control, vestuario, botiquines y la nave, que lindaba con el edificio $B$, que se destinaba a taller mecánico. En la segunda planta se ubicaba el taller de chapa, el almacén de chapa y los comedores de obreros. El edificio A tenía una anchura de cinco módulos, es decir, treinta y dos metros.

El edificio B consta de cinco plantas. La primera (planta baja) se destinaba a acceso principal de los coches a la Filial, a líneas de aceptación, a estación de servicio, a diagnosis y a oficinas del Servicio de Asistencia Técnica, mientras en la segunda planta (planta primera), se ubicaba la línea de cupones y el depósito de coches revisados. En la tercera (planta segunda), se ubicaban las líneas de puesta a punto y el depósito de coches listos para la entrega y, por último, las plantas cuarta y quinta estaban destinadas exclusivamente a depósito de coches nuevos. Todas estas plantas quedaban comunicadas entre sí por rampas, un monta-coches, una escalera y un ascensor. Este edificio, además, contaba con una planta sótano destinada a servicios de acondicionamiento, grupo electrógeno y máquinas de aparatos elevadores, que no ocupaba más de tres módulos de la de la zona lindante con el edificio $\mathrm{A}$.

El edificio $C$, de una sola planta, se destinaba a almacén de recambios con sus correspondientes oficinas. El D, por su parte, estaba compuesto por dos plantas. La primera (o baja) se destinaba a acceso de personal de oficinas, vestuarios de empleados, oficinas de venta y depósito de coches para entrega inmediata y la segunda para oficinas. Por último, el edificio $\mathrm{E}$ se destinaba a exposición y estaba unido directamente al edificio $\mathrm{B}$ y $\mathrm{D}^{5}$ (Fig. 11).

Las fachadas en este proyecto tienen gran importancia ya que son las señas de identidad, la imagen de modernidad que pretende proyectar la marca. Además, se diseñan y ejecutan perfectamente según su orientación y

\footnotetext{
${ }^{5}$ Memoria de proyecto de Filial de S.E.A.T. en Valencia. Visado el 17 de julio de 1965.
} 
pertenencia a un bloque determinado del conjunto, utilizando recursos ya ensayados por el arquitecto en su proyecto de planta de embotellar Coca-Cola o del Colegio de La Pureza, cercano al edificio Seat, como son por ejemplo las marquesinas horizontales en la fachada sur del edificio D.

Al igual que las plantas, todas las fachadas se trabajaron siguiendo el módulo Seat de seis con cuarenta metros, teniendo así una correspondencia total con la estructura del edificio, lo que hacía que fuera un conjunto completamente armónico. Este módulo, o la división del mismo en partes iguales, se dejaba ver en las carpinterías, en los muros cortina y también en los bastidores de los brise-soleil de la fachada del edificio B. Por tanto, era un módulo que acompañaba la fachada en su totalidad y quedaba bien remarcado en los planos de alzado y detalle.
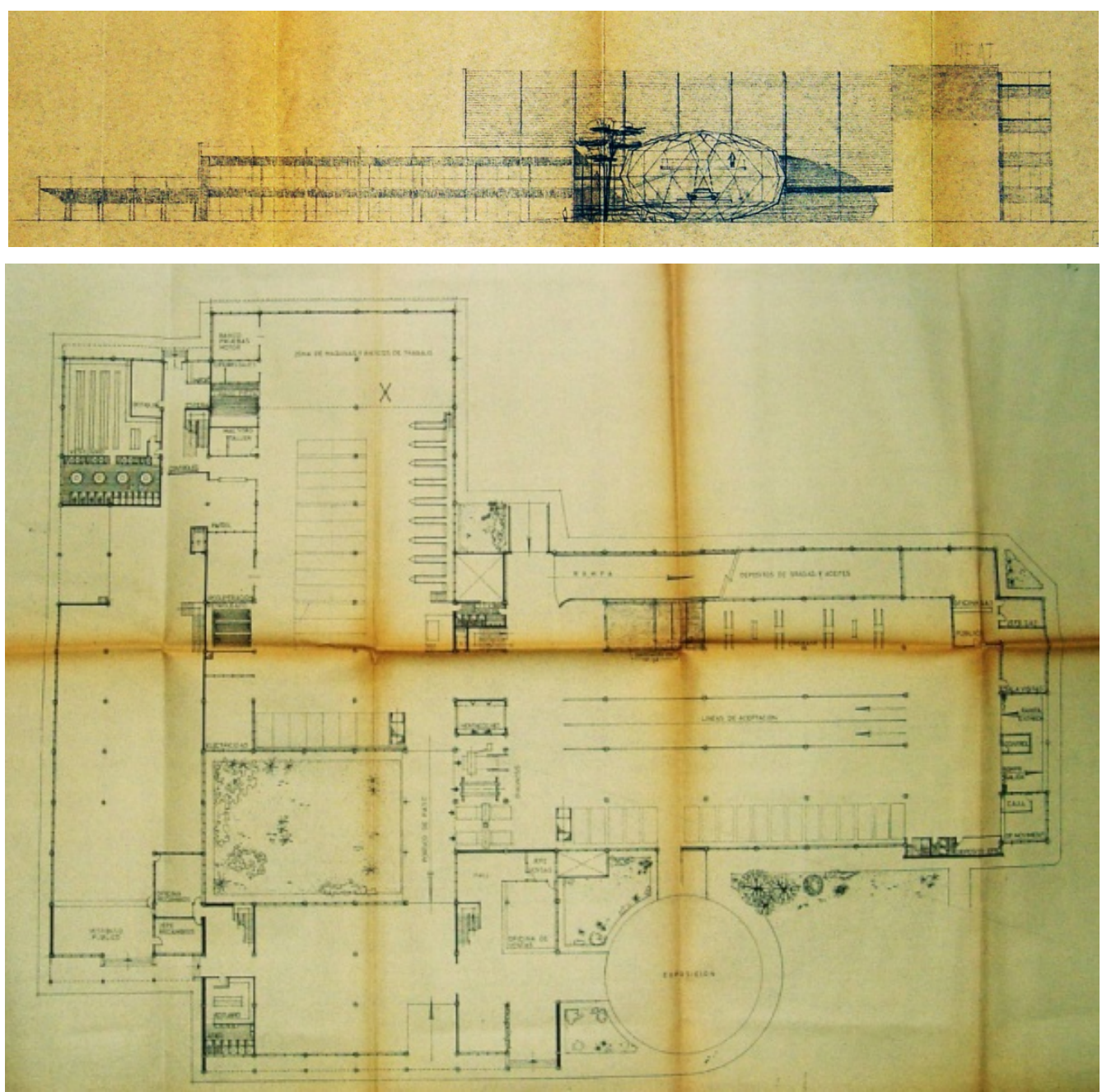

Fig. 11. Alzado principal del anteproyecto Filial Seat en Valencia de marzo de 1964 y plano $n^{\circ} 4$, planta baja del proyecto de edificio para la filial Seat de Valencia de mayo de 1965.

El edificio debía ser construido en el menor tiempo posible lo que motivó, entre otros detalles, la utilización de estructura metálica y el máximo uso de elementos prefabricados en fachada, a parte de los que hacían referencia al tipo de construcción local, razón por la cual aparecían grandes paños de ladrillo caravista o aplacados con piedra artificial blanca. Entre los materiales prefabricados que Mauro Lleó utilizó contó con paneles de Glasal, Ytong y carpinterías y brise-soleil de la empresa CASA, lo que plasmó en sus planos de proyecto.

El Glasal se utilizó como parte de un panel sandwich de la empresa PANASA que formó mediante Glasal blanco, aislante y Glasal azul en su cara exterior. Con un espesor total de treinta y ocho milímetros y paños de uno con veinte por uno con cincuenta metros aproximadamente, este se colocó en parte de los cerramientos de la nave y almacén de recambios.

Por su lado, el Brise-Soleil de la empresa CASA consistía en paneles de rejilla de aluminio que combinaban un 
control eficaz de la luz solar con una amplia flexibilidad en el diseño, aspecto y acabado. Sus aletas de diseño especial, montadas en ranuras inclinadas sobre perfiles, presentaban la ventaja de controlar de una manera eficaz la energía solar. Los perfiles eran extruidos en aleación de aluminio de alta calidad, así como las chapas para la fabricación de las aletas y los elementos de sujeción eran de aluminio y acero inoxidable para impedir su corrosión. Aunque se permitía su montaje de tres formas, paneles apoyados, en voladizo o suspendidos, en la filial se montaron los paneles suspendidos de una estructura auxiliar directamente apoyada en la estructura del edificio.

CASA también diseñó la carpintería del muro cortina de la fachada norte del edificio de depósito de coches que adquirió con esta el carácter de escaparate de automóviles. Así, por la noche, cuando el edificio encendía sus luces, dejaba ver los vehículos nuevos de su interior, en varias plantas, actuando como reclamo para el posible comprador. El modelo utilizado para solucionar esta fachada fue el S-45 de la serie Arcadia. Los paños entre la carpintería se resolvieron con vidrio fijo y, en el caso de haber aberturas, estas eran correderas (Fig. 12).

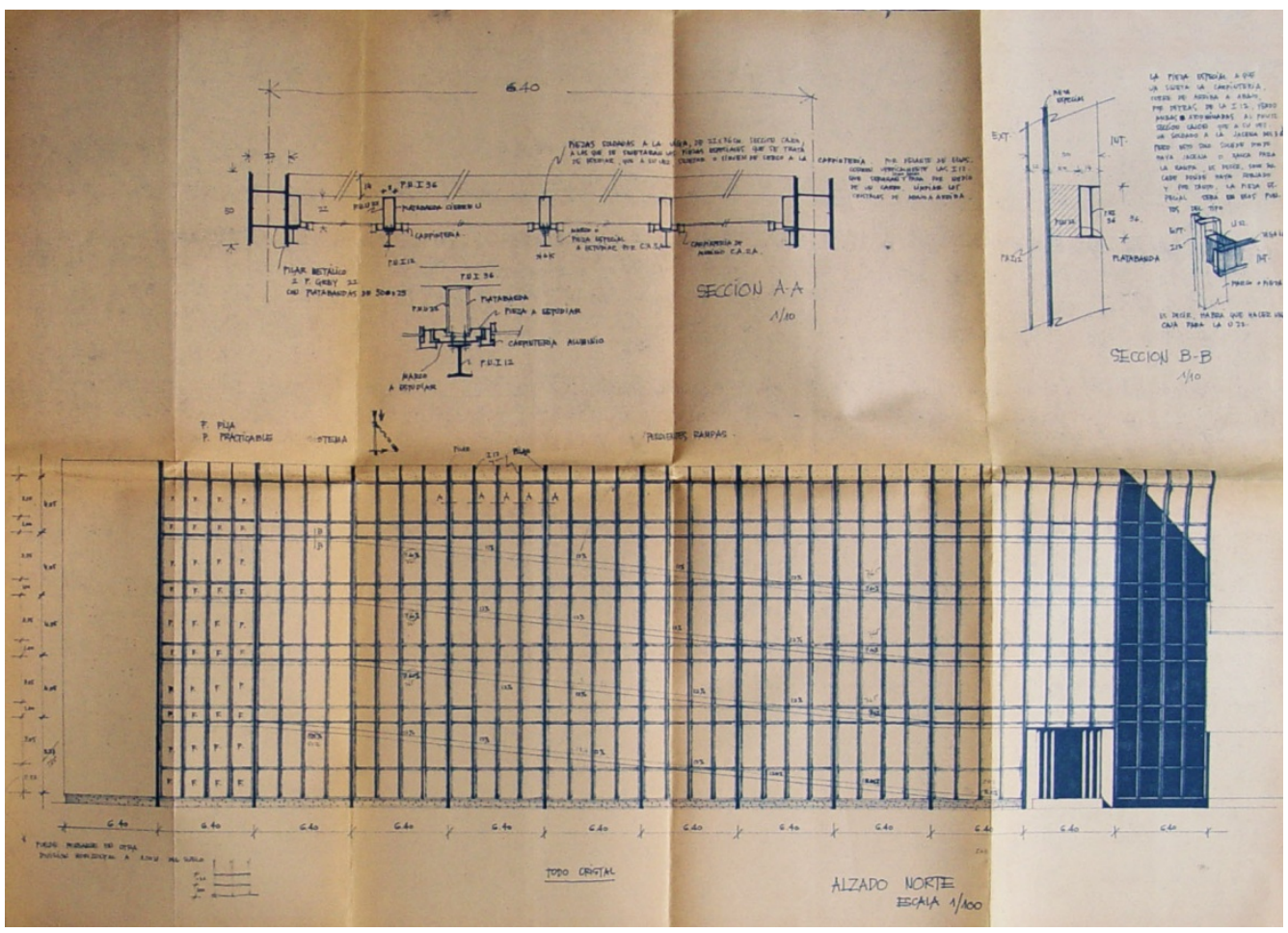

Fig. 12. Detalle fachada norte edificio B del proyecto de edificio para la filial Seat de Valencia. Mayo de 1965.

\section{Conclusión}

Tres edificios industriales, todos ellos obra del mismo arquitecto, Mauro Lleó Serret, consiguieron gracias al acierto en su composición, ejecución y materialidad, dar un paso adelante hacia la modernidad en la ciudad de Valencia.

Los tres dividían su programa en bloques paralelepipédicos distintos que, yuxtapuestos o maclados, solucionaban los distintos espacios y los relacionaban. Además, cada uno de ellos supuso un paso hacia la construcción prefabricada y estandarizada que en el caso de Mauro Lleó tuvo su mejor ejemplo en la filial Seat. Así, poco a poco, sus propuestas se iban acercando a la de los grandes maestros como Mies, que los arquitectos locales conocían gracias a publicaciones nacionales e internacionales y ahora podían ponerlas en práctica gracias a la situación política y económica del país.

El primero de estos edificio, la planta de embotellar Coca-Cola en Quart de Poblet (1958), aún siendo deudor de los modelos de Gutiérrez-Soto en cuanto a materiales de acabado como el ladrillo caravista y el gresite, juega en su composición con volúmenes sencillos de una o dos plantas que macla con acierto relacionando el programa que contiene cada uno de ellos. Cabe mencionar la solución de cubierta en diente de sierra de la nave de alma- 
cenamiento que será uno de los recursos compositivos que caracterizará casi todos los proyectos industriales del arquitecto.

El siguiente paso corresponde al Proyecto de edificio para fábrica de transformados metálicos Flex en Quart de Poblet (1961). Este edificio industrial en altura muestra en fachada la retícula estructural que lo sostiene que, aunque todavía de hormigón, se confirma como recurso compositivo que va acercando al arquitecto a las soluciones constructivas miesianas. De nuevo tres volúmenes sencillos, en este caso yuxtapuestos, forman el conjunto, siendo su fachada principal, la sur, la que con su retranqueo crea un juego de sombras al mismo tiempo que la protege del soleamiento mediante una marquesina formada por elementos lineales prefabricados de hormigón.

Por último, el Proyecto de filial de Seat en Valencia (1965), constituirá un paso hacia la construcción estandarizada y la prefabricación, aunque todavía con recelos, en la solución de sus fachadas a base de muros cortina, brise-soleil y marquesinas metálicas. Compositivamente el edificio responderá al módulo de seis con cuarenta metros que la empresa marca como premisa para todos sus proyectos, distribuyendo alrededor de un patio interior cinco edificios que resuelven cada uno de ellos con su altura y dimensión una parte del programa. Entre estos, destacar volumétricamente el edificio de taller, por la solución de su cubierta en diente de sierra, y el edificio de exposición de vehículos, una esfera geodésica de acero y vidrio, que finalmente no se construyó, y que recuerda a las diseñadas por Buckminster Fuller.

Pero cabe decir que estos edificios no están aislados y comparten soluciones con otros en los que en ocasiones coincide el promotor, son cercanos en cuanto a su situación o conocen por haber colaborado en otros edificios con sus proyectistas.

En el caso de la filial de Seat podemos relacionarla directamente con la solución de fachada de los laboratorios Seat de Barcelona (1958-1960) de César Órtiz Echagüe y Rafael Echaide Itarte, a base de una fachada de muro cortina o con la solución del edificio industrial Frutagut de Oliva (1960), obra de Luis Jiménez de la Iglesia, de estructura metálica y cubierta en diente de sierra de su nave de dos plantas. En el caso del edificio Flex, la relación no es tan directa pero podría relacionarse con la sede del Diario Arriba (1960-1963) de Francisco de Asís Cabrero con el que colaboró en un proyecto de pabellón para la Cooperativa Nacional del arroz en la I Feria Internacional del Campo de 1953. Este conjunto divide su programa en un edificio en altura, de oficinas, y una nave de talleres, al igual que hace Mauro en el edificio Flex. Sin embargo, en el edificio Flex el edificio en altura se destina a fabricación, pues se trata de trabajos de poco peso y la nave sirve únicamente para almacenamiento y talleres. Ambos con la estructura en fachada, resuelven los paños entre esta con ladrillo caravista, sin embrago, Mauro Lleó construirá con hormigón la estructura por razones de seguridad mientras que de Asís Cabrero podrá utilizar ya la estructura metálica.

En el edificio de Coca-Cola, la situación se invierte, sirviendo en este caso la planta valenciana como referente para otras como la de Barcelona (1961-1963) de Josep Soteres i Maruri, que parte de la premisa de mostrar el proceso de fabricación a los transeúntes y ordena la planta en forma de L, tal y como lo hicieran Lleó y Albert.

\section{Bibliografía}

ALONSO DE ARMIÑO PÉREZ, L., JORDÁ SUCH, C. y VIDAL VIDAL, V. M. Fondo Documental del s. XX, Comunitat Valenciana. [CD]. Valencia: Colegio Oficial de Arquitectos de la Comunitat Valenciana, Consejería de Obras Públicas, Urbanismo y Transporte, Instituto Valenciano de la Edificación, 2002 [consulta: agosto de 2014].

AA.VV. Equipamientos I. Lugares públicos y nuevos programas, 1925-1965. Registro DO.CO.MO.MO. Ibérico. Barcelona: Fundación Caja de Arquitectos, Fundación DO.CO.MO.MO. Ibérico, 2010. 414 p. ISBN: 9788493785734.

AA.VV. Equipamientos II. Ocio, deporte, comercio, transporte y turismo. Registro DO.CO.MO.MO. Ibérico, 19251965. Barcelona: Fundación Caja de Arquitectos, Fundación DO.CO.MO.MO. Ibérico, 2011. 80 p. ISBN: 9788493785772.

BRAVO, R. y CANOVAS, A. "La arquitectura de la Comunitat Valenciana”. Arquitectos. 1991, pp.120.

CALDUCH CERVERA, J. "El declive de la arquitectura moderna: deterioro, obsolescencia, ruina". Palapa. 2009, vol. IV, núm. II.

COSTA, X., LANDROVE, S.(dir). Arquitectura del Movimiento Moderno. Registro DO.CO.MO.MO. Ibérico, 1925- 
1965. Barcelona: Fundación Mies van der Rohe y DO.CO.MO.MO. Ibérico, 1991. 336 p. ISBN: 84-89698-22-8.

GAJA DÍAZ, F. y BOIRA MAIQUES J. V. "Planeamiento y realidad urbana en la ciudad de Valencia (1939-1989)". Cuadernos de Geografía. 1994, 55, p. 63-89.

GARCíA BRAÑA, C., LANDROVE, S., TOSTOES, A. La arquitectura de la industria, 1925-1965. Registro DO.CO.MO.MO. Ibérico. Barcelona: Fundación DO.CO.MO.MO. Ibérico, 2005. 275 p. ISBN: 84-609-1196-9.

JORDÁ SUCH, C. 20x20. Siglo XX, veinte obras de arquitectura moderna. València: Colegio Oficial de Arquitectos de la Comunitat Valenciana, Conselleria d'Obres Públiques, Urbanisme i Transports, 1997. 164 p. ISBN: 8486828-18-X

JORDÁ SUCH, C. "Docente y moderna. Crónica del panorama valenciano actual". Arquitectura viva. 2007, pp.103.

JORDÁ SUCH, C., MARTÍNEZ MEDINA, A., PRIOR Y LLOMBART, J. Arquitectura moderna y contemporánea de la Comunitat Valenciana. [CD]. València: Colegio de Arquitectos de la Comunitat Valenciana, Consellería d'Infraestructures, Territori i Medi Ambient, 2012 [consulta: agosto de 2014].

LLEÓ SERRET, M. “El arte y el planeamiento urbano". Archivo de Arte Valenciano. 1971.

MARTíN ACEÑA, P. y COMÍN COMÍN, F. "La acción regional del Instituto Nacional de Industria". En: NADAL OLLER J., CARRERAS I ODRIOZOLA A. (dir.) Pautas regionales de la industrialización española: siglos XIX y XX. Barcelona: Ariel, 1990. p. 379-420.

La avenida de Castilla se abre al tránsito. Las Provincias 1953 [en línea] valenpedia.lasprovincias.es [consulta: 10 de agosto 2014]. Disponible en:

http://valenpedia.lasprovincias.es/historia-valencia/1953/la avenida de castilla se abre al transito

REIG MARTÍNEZ, E. "Historia breve de la industria valenciana". En: Dos siglos de industrialización en la Comunitat Valenciana. Valencia: Colegio Oficial de Ingenieros Superiores Industriales de la Comunitat Valenciana, 2007. p. 27-63. 


\section{Biografía}

Carmen Martínez Gregori. Arquitecta (2003) por la Universidad Politécnica de València (UPV). Obtiene el Diploma de Estudios Avanzados (2007) en el Departamento de Urbanismo de la UPV y un año más tarde empieza a ejercer como profesora asociada en el Departamento de Composición Arquitectónica de la misma universidad (2008) donde obtiene el titulo de Doctora (2015) con la tesis titulada "De la autarquía a la modernidad: la obra de Mauro Lleó".

Participa en diversos trabajos de investigación dependientes de la Consellería de Medio Ambiente, Agua, Urbanismo y Vivienda, como "Taller V: H+l, Vivienda, Habitabilidad e Innovación" (2006), y del Colegio Oficial de Arquitectos de la Comunidad Valenciana (COACV), como el "Projecte Terra" (2008) o "Arquitectura Moderna y Contemporánea de la Comunitat Valenciana" (2012).

Desde 2003 trabaja como arquitecta por cuenta propia obteniendo varios reconocimientos, entre otros, de ASCER, del IVVSA, del IBAVI y del COACV, siendo estos publicados en revistas de ámbito nacional como Arquitectos, TC Cuadernos de Arquitectura, Temas de Arquitectura o Detail.

\section{Biography}

Carmen Martinez Gregori. Architect (2003) from the Polytechnic University of Valencia (UPV). Gets the Diploma of Advanced Studies (2007) at the Department of Urban Planning at the UPV and a year later began to work as an associate professor in the Department of Architectural Composition at the same university (2008) where she obtained a doctorate degree (2015) with the thesis entitled "From autarky to modernity: the work of Mauro Lleó".

Participates in various research jobs dependent of the Department of Environment, Water, Urbanism and Housing, as "Workshop V: H + I, Housing, Livability and Innovation" (2006), and the Official College of Architects of Valencia (COACV), as the "Projecte Terra" (2008) or "Modern and Contemporary Architecture of Valencia" (2012).

Since 2003 works as a self-employed architect obtaining several awards, among others from ASCER, the IVVSA, the IBAVI and COACV, these being published in national magazines such as Architects, TC Journal of Architecture, Architecture Themes or Detail. 